\title{
Some Effects on Fatigue Strength
}

It has been widely known for some time that the mean stress affects the fatigue strength of steel. This is distinctly evident when working with non-welded or stress-relieved welded details. Some consequences of this influence are revealed when evaluating the stress history, or when counting the cycles.

In this paper, decisive influences on the plain fatigue limits or strengths are indicated, when applying a mean stress, together with the effects of both combined stress and anisotropy and the minimum stress required to grow a crack of a given length and depth.

Keywords: alternating stress, mean load, notched and cracked specimen, notched fatigue data, reversed direct stress, stress ratio.

\section{Introduction}

When the plain fatigue limit or the plain fatigue strength of a material is determined from a series of specimens tested at zero mean load, it is of practical importance to clarify how this value is influenced by, for instance, a superimposed mean load, or a combined stress loading. It may be necessary to know the effect of a combination of these variables. This information has to be assessed from a careful observation of the variables acting separately.

There is no recent evidence to suggest that compressive mean stresses reduce the zero mean stress fatigue limit. The data suggests that the fatigue limit either remains constant or increases, approximately linearly, above the zero mean stress value as the compressive mean stress increases in magnitude, provided that buckling or gross yielding does not occur.

The initiation of a surface microcrack in a wrought ductile metal depends on the resolved cyclic shear stresses necessary to cause continuing cyclic slip exceeding some minimum value. Therefore, the effect of the mean stress on its fatigue limit depends on the extent to which this minimum value is either increased or reduced by the resolved static stresses acting both along and normal to the operative slip planes. The data implies that, for metals exhibiting a sharp knee and a definite fatigue limit, this effect is small until the maximum stress in the cycle is above the yield stress; for other materials there is a gradual decrease with increasing static tensile stress and perhaps a slight increase with increasing compressive mean stress.

\section{Influence of uniaxial loading}

The stress cycle $\sigma_{m} \pm \sigma$, where $\sigma_{m}$ is considered positive, is demonstrated in Fig. 1; it follows that

$$
\begin{aligned}
& \sigma_{\text {max }}=\sigma_{m}+\sigma \\
& \sigma_{\text {max }}=\sigma_{m}-\sigma .
\end{aligned}
$$

The ratio $\sigma_{\min } / \sigma_{\max }$ is called the stress ratio and is commonly denoted by $R$.

Algorithms to study the effect of mean stress generally involve establishing $\mathrm{S} / \mathrm{N}$ curves for a series of values of mean stresses, so that a diagram can be plotted showing the relationship between the fatigue limit at a particular mean stress and the corresponding value of the mean stress. In order to avoid the necessity of carrying out comprehensive series of tests at different mean stresses, on different materials, attempts have been made to formulate relationships linking the

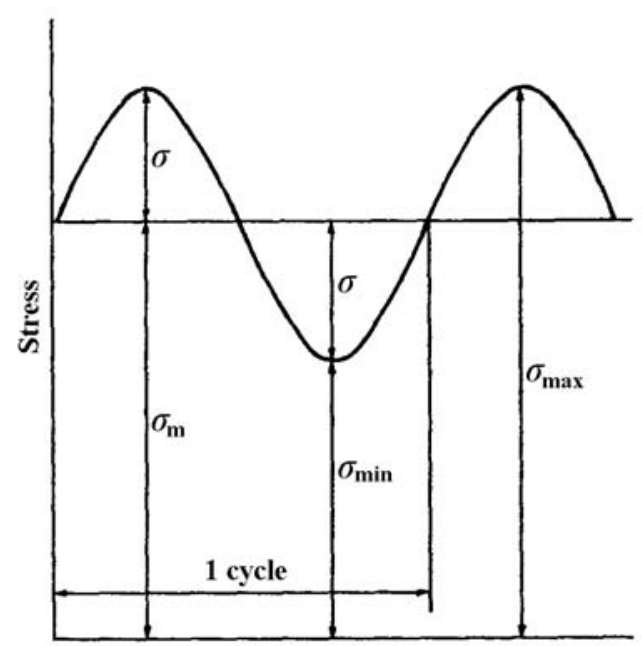

Fig. 1: Tensile loading cycle

pertinent variables, thus enabling the fatigue limit of a material (or the strength at a given endurance) under a given mean stress to be predicted from the fatigue limit at zero mean stress. The most common requirement for practical design purposes is the fatigue limit under a tensile mean stress. According to [1] the two relationships are generally accepted as representing the experimental data, one due to Goodman and the other due to Gerber, which found that Wöhler's tensile mean stress data conformed to a parabola, having as end-points the fatigue limit at zero mean stress and the tensile strength of the material. Goodman assumed that the safe repeated tension loading cycle was zero to half the tensile strength of the material $R_{m}$, that is, $R_{m} / 4 \pm R_{m} / 4$, the safe stress decreasing linearly to zero at $R_{m}$ and increasing linearly to the zero mean stress condition; this gives a zero mean stress fatigue limit of $\pm R_{m} / 3$. This relationship was subsequently modified (and is now called the modified Goodman relationship) so that the fatigue limit decreased linearly with increasing tensile mean stress from its experimentally determined zero mean stress value to zero at $R_{m}$, the tensile strength of the material. The two relationships are illustrated in Fig. 2; they can be expressed in the form

$$
\pm \sigma= \pm \sigma_{c}\left\{1-\left(\frac{\sigma_{m}}{R_{m}}\right)^{n}\right\},
$$




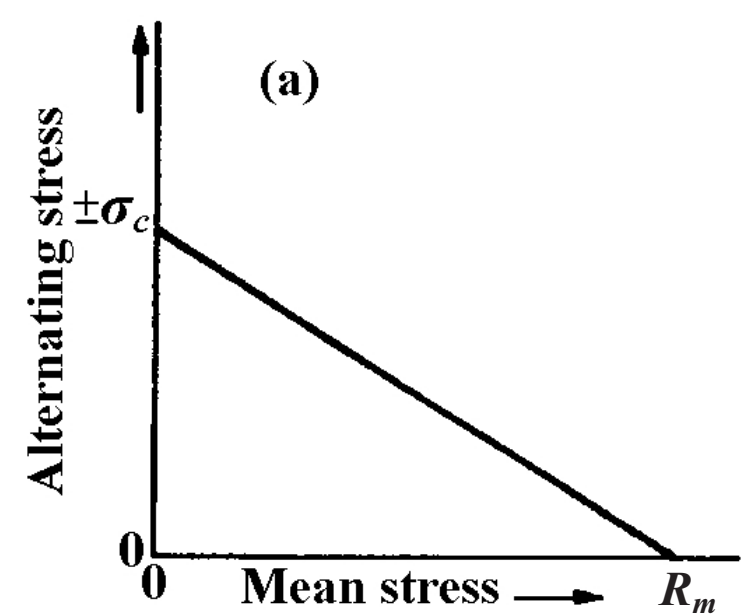

Fig. 2: Modified Goodman (a) and Gerber (b) diagrams

where $\pm \sigma$ is the fatigue limit (or strength at a given endurance) when a tensile mean stress sm is present, $\pm \sigma_{c}$ is the fatigue limit (or strength at the same endurance) at zero mean stress, $R_{m}$ is the tensile strength, $n=1$ is the modified Goodman relationship, and $n=2$ is the Gerber relationship.

If the above equation is written in the form (omitting \pm signs)

$$
\frac{\sigma}{\sigma_{c}}=1-\left(\frac{\sigma_{m}}{R_{m}}\right)^{n},
$$

the diagram of Fig. 2 can be replotted using the non-dimensional variables $\sigma / \sigma_{c}$ and $\sigma_{m} / R_{m}$ as shown on Fig. 3. This figure is often referred to as the R-M diagram because it shows the relationship between the safe range of stress $R$ and the mean stress $M$. If a line is drawn in Fig. 3 joining points $\mathrm{A}$ and $\mathrm{B}$, where $\mathrm{A}$ and $\mathrm{B}$ are the yield of the material divided by $\sigma_{c}$ and $R_{m}$ respectively, then points to the right of $\mathrm{AB}$ represent tests in which the maximum tensile stress in the cycle is sufficient to cause gross yielding. To ensure that neither yielding nor fatigue failure occurs, a diagram similar in form to the modified Goodman diagram has been proposed in which

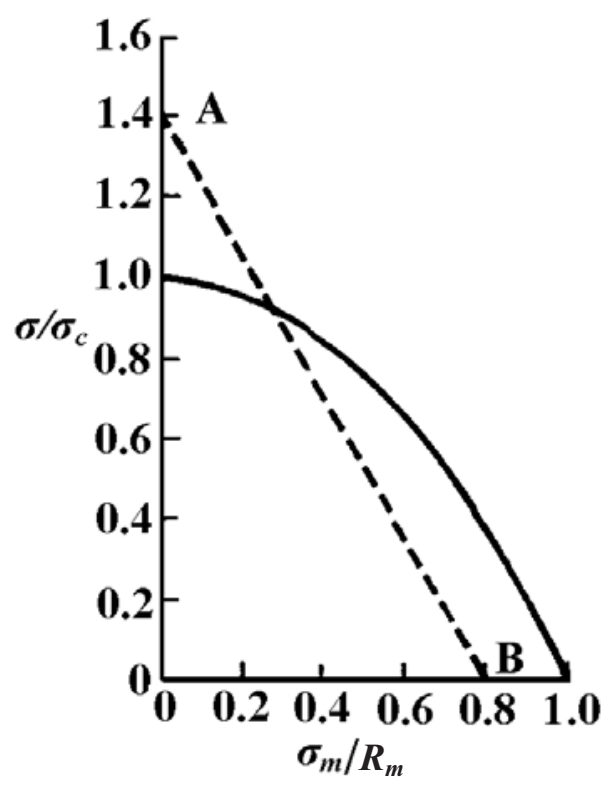

Fig. 3: R-M diagram

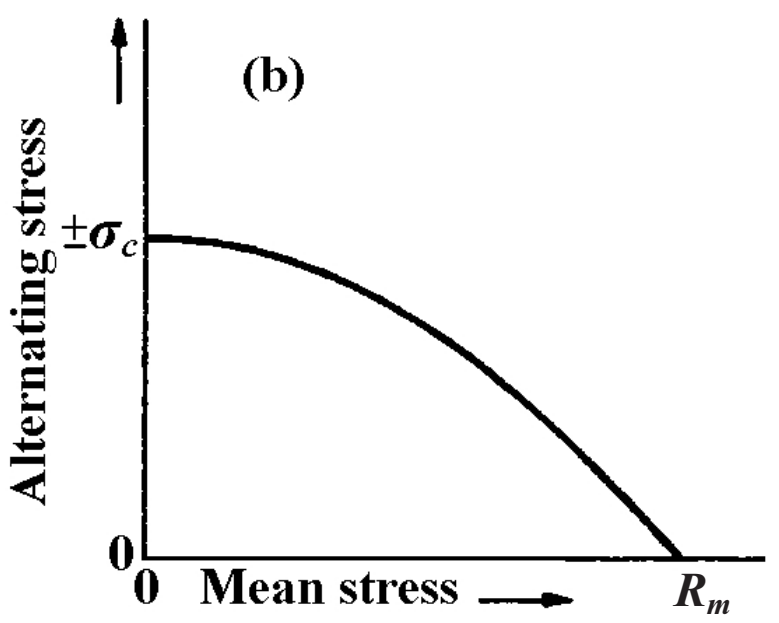

the criterion of failure at zero alternating stress is taken as the yield instead of the tensile strength; the straight line joining this point to $\pm \sigma_{c}$ is often referred to as the Soderberg line. In all these diagrams, loading conditions inside the curve or straight line are supposedly safe, while those outside lead to failure.

The experimental data shows, that the fatigue limit tends to increase above the zero mean stress value with increasing compressive stress, provided the specimen does not yield or buckle.

Heywood [2] derived an empirical relationship from an analysis of available data which can be written in the form

$$
\frac{\sigma}{R_{m}}=1-\left(\frac{\sigma_{m}}{R_{m}}\right)\left\{\frac{\sigma_{c}}{R_{m}}+\gamma\left(1-\frac{\sigma_{c}}{R_{m}}\right)\right\},
$$

where

$$
\gamma=\left(\frac{\sigma_{m}}{3 R_{m}}\right)\left(2+\frac{\sigma_{m}}{R_{m}}\right) \text { for steels }
$$

and

$$
\gamma=\left(\frac{\sigma_{m}}{R_{m}}\right)\left\{1+\left(\frac{n R_{m}}{2200}\right)^{4}\right\}^{-1} \text { for aluminium alloys. }
$$

$n$ is the logarithm of the life at which the fatigue strength is estimated and all stresses are in MPa, $g$ can be considered as the curvature factor of a line on the $\sigma / \sigma_{c}$ versus $\sigma_{m} / R_{m}$ diagram, the expression reducing to the modified Goodman relationship (2) when $\gamma=0$.

Some experimental data relating to the effect of a mean stress on the uniaxial (reversed direct stress) fatigue limit of various steels are given below, according to [3], in Table 1.

Data obtained on wrought metallic alloys (steels, aluminium alloys) subjected to tensile mean stresses tends to lie between the modified Goodman and Gerber lines. A survey of the literature showed that 90 percent of the data lay above the Goodman line, falling mainly between the Goodman and Gerber lines. However, some of the low- and medium-strength aluminium alloys give values lying below the Goodman line.

Provided a specimen does not yield or buckle under the maximum compressive stress in the loading cycle, the fatigue 
Table 1:

\begin{tabular}{|c|c|c|c|c|c|}
\hline \multirow{3}{*}{ Material } & \multirow{3}{*}{$\begin{array}{c}\text { Tensile } \\
\text { strength } \\
(\mathrm{MPa})\end{array}$} & \multirow{3}{*}{$\begin{array}{l}\text { Tensile mean stress } \\
\qquad(\mathrm{MPa})\end{array}$} & \multicolumn{3}{|c|}{ Fatigue strength (MPa) } \\
\hline & & & \multicolumn{2}{|c|}{ Calculated } & \multirow[t]{2}{*}{ Experimental } \\
\hline & & & Modified Goodman & Gerber & \\
\hline \multirow{7}{*}{ Mild steel } & \multirow{7}{*}{410} & & & & fatigue limit \\
\hline & & 0 & \pm 190 & \pm 190 & \pm 190 \\
\hline & & 43 & \pm 160 & \pm 185 & \pm 182 \\
\hline & & 91 & \pm 148 & \pm 179 & \pm 168 \\
\hline & & 148 & \pm 122 & \pm 165 & \pm 159 \\
\hline & & 250 & \pm 74 & \pm 120 & \pm 153 \\
\hline & & 310 & \pm 46 & \pm 82 & \pm 105 \\
\hline \multirow{5}{*}{ Nickel-chromium alloy steel } & \multirow{5}{*}{865} & & & & fatigue limit \\
\hline & & 0 & \pm 480 & \pm 480 & \pm 480 \\
\hline & & 155 & \pm 394 & \pm 465 & \pm 448 \\
\hline & & 310 & \pm 310 & \pm 418 & \pm 418 \\
\hline & & 465 & \pm 224 & \pm 340 & \pm 356 \\
\hline \multirow{5}{*}{15130 steel } & \multirow{5}{*}{800} & & & & fatigue limit \\
\hline & & 0 & \pm 338 & \pm 338 & \pm 338 \\
\hline & & 70 & \pm 310 & \pm 334 & \pm 338 \\
\hline & & 140 & \pm 283 & \pm 328 & \pm 330 \\
\hline & & 210 & \pm 252 & \pm 317 & \pm 317 \\
\hline
\end{tabular}

limit does not decrease below the zero mean stress value when a compressive mean stress is superimposed. Data on steels and aluminium alloys have been given which imply that the fatigue limit increases linearly with increasing compressive mean stress, the value of the fatigue limit at a compressive mean stress equal to the yield stress of the material being about 1.4 times that at zero mean stress. Some data illustrating the variation of fatigue strength with compressive mean stress is regiven in conformity with [3] in Table 2.

In all cases, it is seen that the fatigue limit either increases above or remains equal to the zero mean stress value. Speci-

Table 2:

\begin{tabular}{|l|c|c|}
\hline Material & $\begin{array}{c}\text { Compressive } \\
\text { mean stress } \\
(\mathrm{MPa})\end{array}$ & $\begin{array}{c}\text { Fatigue } \\
\text { strength } \\
(\mathrm{MPa})\end{array}$ \\
\hline \multirow{3}{*}{ Nickel-chromium alloy steel } & 0 & fatigue limit \\
& -155 & \pm 480 \\
& -310 & \pm 510 \\
\hline \multirow{3}{*}{11 140 steel } & & fatigue limit \\
& 0 & \pm 390 \\
& -173 & \pm 410 \\
\hline
\end{tabular}

mens subjected to a wholly compressive loading cycle exhibited numerous surface cracks, pieces of material often flaking away from the surface.

It has been reported that a more accurate prediction of the effect of a tensile mean stress can be obtained by using true stress instead of nominal stress and the fracture stress of the material instead of the tensile strength. Experimental points from various steels and aluminium alloys tested at various tensile mean stresses fall around a straight line on a true stress/modified Goodman diagram.

A material whose fatigue limit depends on whether or not cracks can grow directly from inherent flaws responds more markedly to mean stress (either tensile or compressive) than a material whose fatigue limit depends on whether or not the applied cyclic stress is sufficient to initiate and develop surface microcracks. Cast iron is an example of the former material, and it is found [3] that a tensile mean stress reduces and a compressive mean stress increases its fatigue limit by a greater extent than that predicted by the modified Goodman relationship (2). The ratio of the fatigue limit of cast iron in repeated compression to that in repeated tension averages about 3.3 compared to an average value of about 1.5 for malleable cast irons and wrought steels. This is further illustrated by the following fatigue limits for grey cast iron [2]:

$$
\begin{array}{ll}
\text { pulsating tension } & 0 \text { to } 100 \mathrm{MPa}, \\
\text { zero mean stress } & \pm 73 \mathrm{MPa}, \\
\text { pulsating compression } & 0 \text { to }-450 \mathrm{MPa} .
\end{array}
$$




\section{Anisotropy and combined stress effects}

In many applications, components are subjected to more general loading cycles, for example, combined bending and torsional loads. Combined cyclic loading comprises either in-phase bending and torsion or in-phase biaxial tension.

Attempts to predict the fatigue limit (or strength at long endurances) at zero mean stress, under a combined stress loading, from the corresponding uniaxial fatigue limit or strength have been based on the usually accepted criteria for predicting the onset of plastic deformation under a static combined stress loading, the limiting static stresses merely being replaced by the corresponding limiting cyclic stress amplitudes. Only biaxial stress systems need be considered in the case of those materials in which cracks are initiated at a free surface and the three failure criteria most commonly used (that is, maximum principal stress, maximum shear stress, and the maximum shear - strain energy or von Mises) may be expressed by the formulae:

maximum principal stress

$$
\sigma_{e}=\frac{\sigma_{x}+\sigma_{y}}{2}+\frac{1}{2}\left\{\left(\sigma_{x}-\sigma_{y}\right)^{2}+4 \tau_{x y}^{2}\right\}^{\frac{1}{2}},
$$

maximum shear stress

$$
\sigma_{e}=\left\{\left(\sigma_{x}-\sigma_{y}\right)^{2}+4 \tau_{x y}^{2}\right\}^{\frac{1}{2}}
$$

von Mises

$$
\sigma_{e}=\left\{\sigma_{x}^{2}-\sigma_{x} \sigma_{y}+\sigma_{y}^{2}+3 \tau_{x y}^{2}\right\}^{\frac{1}{2}}
$$

where $\sigma_{e}$ is the equivalent principal stress, $\sigma_{x}$ and $\sigma_{y}$ are normal stresses, and $\tau_{x \nu}$ is the shear stress. For a cylindrical bar subjected to combined bending and torsional loads, the formulae for surface stresses become:

maximum principal stress

$$
\sigma_{e}=\frac{1}{2}\left\{\sigma+\left(\sigma^{2}+4 \tau^{2}\right)^{\frac{1}{2}}\right\},
$$

maximum shear stress

$$
\sigma_{e}=\left(\sigma^{2}+4 \tau^{2}\right)^{\frac{1}{2}}
$$

von Mises

$$
\sigma_{e}=\left(\sigma^{2}+3 \tau^{2}\right)^{\frac{1}{2}}
$$

where $\sigma$ is the maximum surface bending stress and $\tau$ is the maximum surface shear stress. Verification of whether or not fatigue data obtained on a particular material conforms to any of these criteria $(5 \mathrm{a}-\mathrm{c})$ may be obtained from the value of the ratio of its torsional fatigue limit to the uniaxial fatigue limit (the above criteria require this ratio equal to $1,0.5$, and 0.577 respectively) and by determining the fatigue limits of specimens subjected to various combined stress loadings. Suitable methods for performing these tests are either to apply in-phase bending and torsional stresses to cylindrical specimens or to subject thin-walled tubes to in-phase pulsating internal pressure and axial cyclic loading.

Table 3:

\begin{tabular}{|l|c|}
\hline \multicolumn{1}{|c|}{ Materials } & $\begin{array}{c}\text { Ratio of fatigue limit in torsion to } \\
\text { that in rotating bending }\end{array}$ \\
\hline 19 carbon steels & 0.55 \\
\hline 14 alloy steels & 0.58 \\
\hline
\end{tabular}

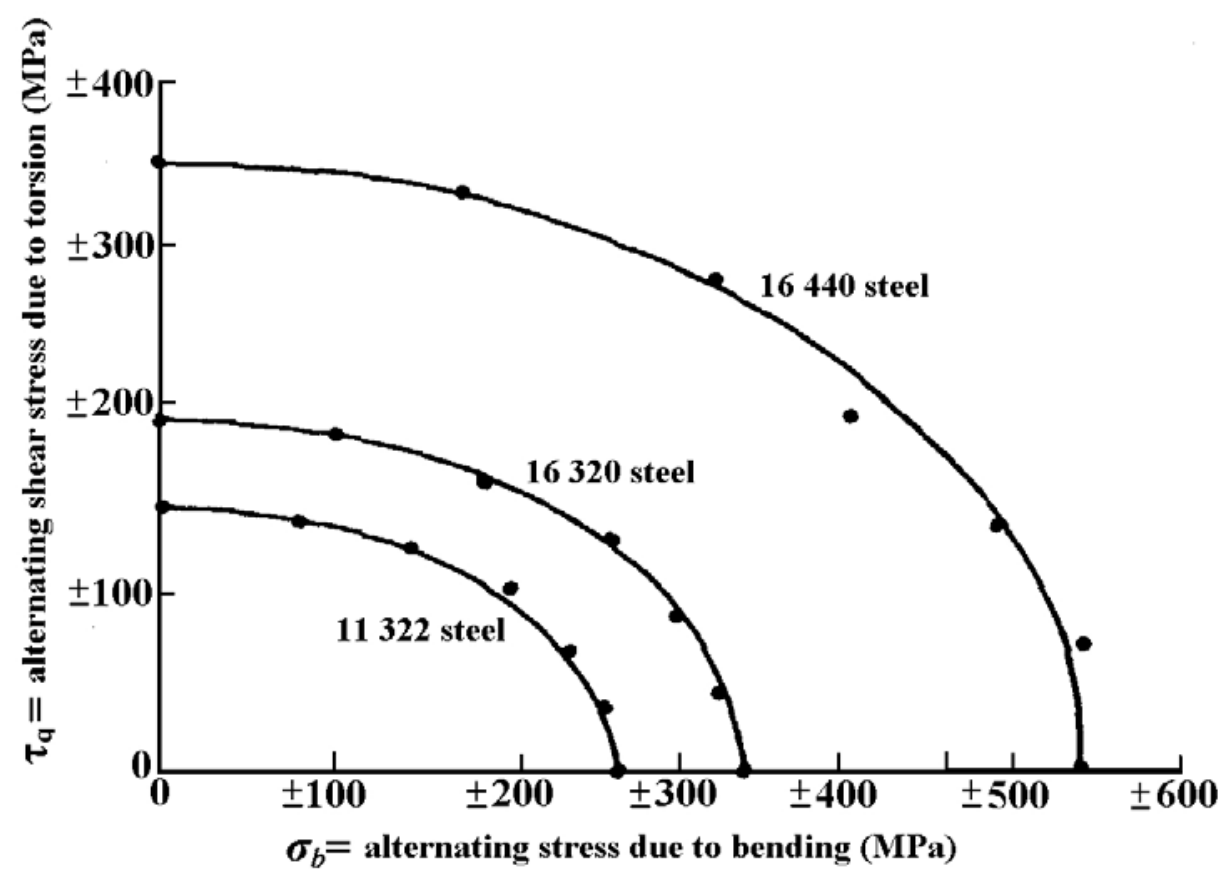

Fig. 4: Fatigue limits of 3 steels under combined bending and torsional alternating stresses (taken from Frost [1]) 
Average values of the ratio of the fatigue limit in torsion to that in rotating bending are demonstrated in Table 3 .

It would appear, therefore, that for metals in which failure is initiated at a free surface, the ratio of the fatigue limit (or strength at very long endurances) in torsion to that in uniaxial loading has an average value not far removed from that predicted by the von Mises criterion, the value for a particular material depending on the endurance at which the fatigue strength is estimated and on a metallurgical structure.

Most data relating to combined stresses were obtained for combined bending and torsional stresses. Machines were designed to apply either an alternating bending or an alternating torsion loading or a loading consisting of any in-phase combination of the two, to obtain data for numerous ferrous alloys. The fatigue limit (zero mean stress) of each material was obtained under alternating bending, alternating torsion, and five different combinations of alternating in-phase bending and torsional stresses, using $7.6 \mathrm{~mm}$ diameter plain specimens. None of the usual theories of failure under combined stresses were found to represent all the results; instead, it was suggested that for the wrought steels tested (these covered a range of tensile strengths from $400 \mathrm{MPa}$ to $1850 \mathrm{MPa}$ ) the experimental results for each steel could be represented by an ellipse quadrant having as end points the fatigue limits in pure torsion and pure bending. Three typical groups of results are shown in Fig. 4.

The equation of the ellipse quadrant is (omitting \pm signs)

$$
\frac{\sigma_{b}^{2}}{\sigma_{c b}^{2}}+\frac{\tau_{q}^{2}}{\tau_{c q}^{2}}=1,
$$

where $\pm \sigma_{c b}$ and $\pm \tau_{c q}$ are the fatigue limits in pure bending and torsion respectively and $\pm \sigma_{b}$ and $\pm \tau_{q}$ are the stress ranges due to bending and torsion respectively at the fatigue limit under a combined stress loading. This is equivalent to the maximum shear stress criterion (5b) if $\sigma_{c b} / \tau_{c q}=2$ and to the von Mises criterion (5c) if $\sigma_{c b} / \tau_{c q}=\sqrt{3}$. Although it was considered that the $\sigma_{c b} / \tau_{c q}$ ratio obtained for the various steels did not agree exactly with either of these two criteria, consideration of all the data for any one steel shows that, in general, they are not far removed from the values predicted by the von Mises criterion. Some data is replotted, using $\sigma_{b}^{2}$ and $\tau_{q}^{2}$ axes, on Fig. 5.

The full line represents the von Mises criterion; the dotted line is the best straight line through the points.

Values quoted in [3], namely of the torsion/uniaxial ratio for various cast irons, usually lie between 0.9 and 1.0 , thus appearing to conform more closely to the maximum principal stress rather than to the von Mises criterion. Fatigue failure of cast iron is associated, however, with the presence of inherent flaws. Some tests carried out on both cast iron plain specimens and wrought steel notched specimens gave results that could not be represented by an ellipse quadrant. Instead the fatigue limits lay around an ellipse arc having the equation
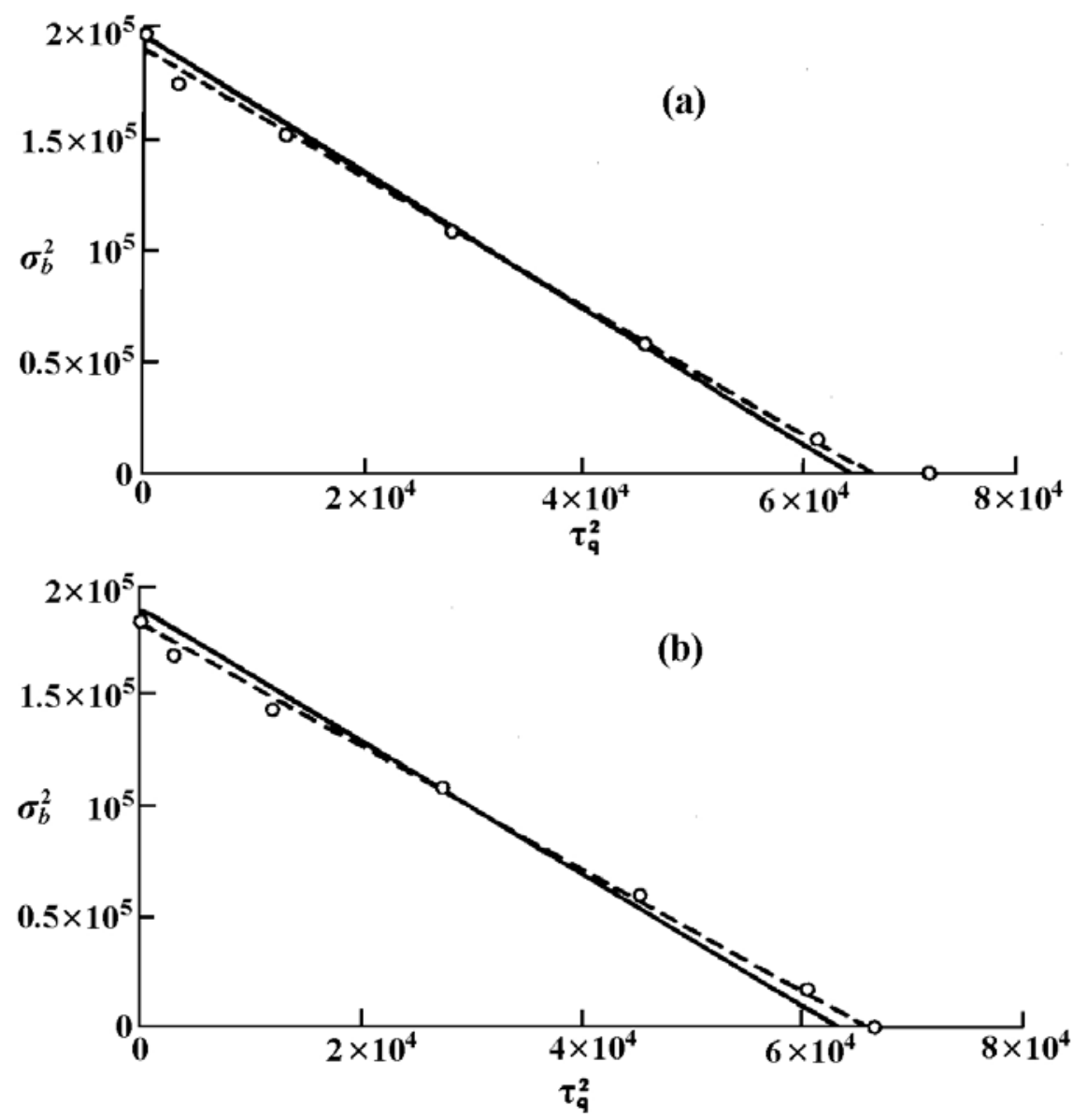

Fig. 5: $\sigma_{b}^{2}$ versus $\tau_{q}^{2}$ diagrams, a) 16320 steel (tensile strength 700-770 MPa), b) Chromium-Vanadium steel (tensile strength 700-770 MPa) (taken from Frost [1]) 
'Silal' cast iron

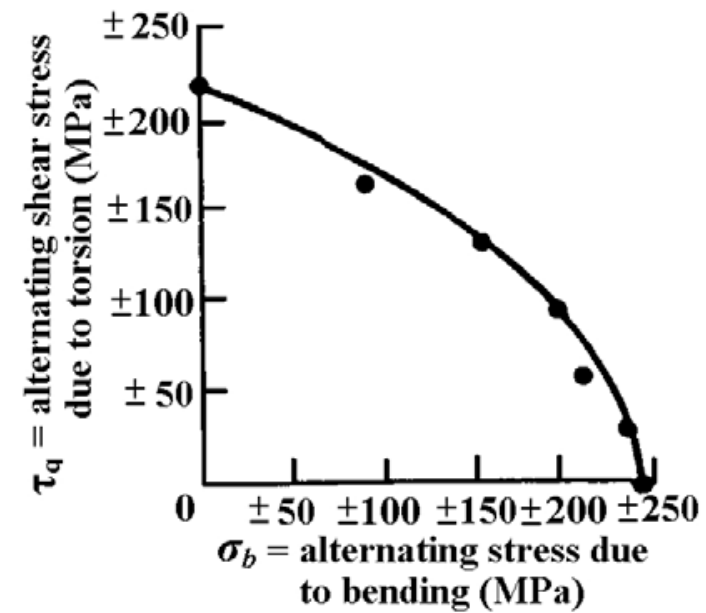

11140 steel notched specimens

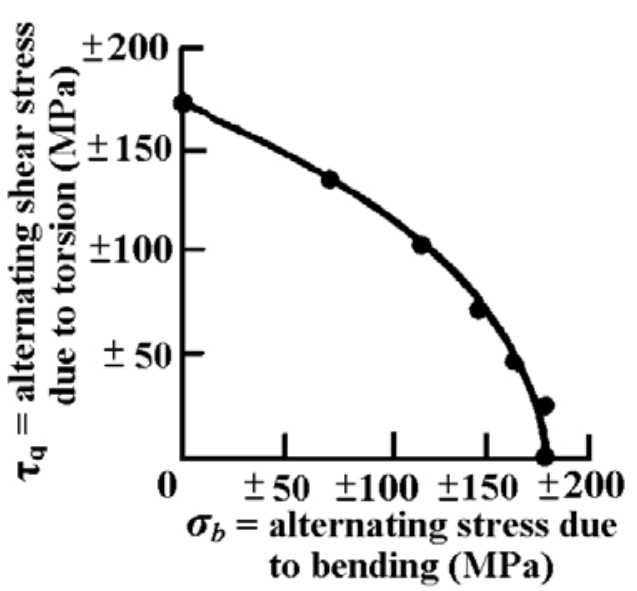

Fig. 6: Fatigue limits of plain cast iron and notched wrought steel specimens under combined bending and torsional alternating stresses (taken from Frost [1])

$$
\frac{\tau_{q}^{2}}{\tau_{c q}^{2}}+\frac{\sigma_{b}^{2}}{\sigma_{c b}^{2}}\left(\frac{\sigma_{c b}}{\tau_{c q}}\right)+\frac{\sigma_{b}}{\sigma_{c b}}\left(2-\frac{\sigma_{c b}}{\tau_{c q}}\right)=1
$$

A set of results obtained from plain "Silal" cast iron specimens and veenotched wrought steel specimens is shown Fig. 6 .

A specimen containing a notch subjected to uniaxial loading has a biaxial stress system set up in the material at the notch root. Tests on chromium-vanadium and 0-14\% C steel specimens, notched to create a known biaxial stress system on the surface of the notch root, gave fatigue limits in good agreement with the von Mises criterion.

The magnitude and sign of the normal stress occurring on the maximum shear stress planes would be expected to influence the initiation and development of surface microcracks [4], and since a normal stress is present on the planes of maximum shear stress in bending but is absent in torsion, it might be expected to produce differences in the ratio of the fatigue limit in bending to that in torsion. To allow for the influence of the normal stress and also for anisotropy of the material, it was suggested that the maximum shear stress criterion, for example, could be modified as follows.

Let $\pm \sigma_{c b}$ and $\pm \tau_{c q}$ fatigue limits in pure bending and torsion, respectively,

$\pm \sigma_{b}$ and $\pm \tau_{q} \quad$ stress ranges due to bending and torsion at the fatigue limit of the combined stress loading,

then, writing $\sigma_{c b}=2 K \tau_{c q}$ (omitting \pm signs), where $K$ is a correction factor to allow for the state of stress and anisotropy, the maximum shear stress criterion can be written as

$$
\sigma_{b}^{2}+4\left(K \tau_{q}\right)^{2}=\sigma_{c b}^{2}
$$

which, on substituting for $K$, gives

$$
\frac{\sigma_{b}^{2}}{\sigma_{c b}^{2}}+\frac{\tau_{q}^{2}}{\tau_{c q}^{2}}=1
$$

Sines [5] suggested that the experimental data could be summarized as follows:

1) the combined stress fatigue limits of wrought metallic alloys were in good agreement with the von Mises criterion,
2) the uniaxial fatigue limit was decreased by a tensile mean stress and increased by a compressive mean stress, the change in fatigue limit, for practical purposes, being linearly dependent on the mean stress, provided the material did not yield,

3) both the torsional and uniaxial fatigue limits were unaffected by a static torsion mean stress, provided the material did not yield,

4) the torsional fatigue limit was affected by a mean tensile or compressive mean stress as in 2),

and thus expressed the relationship between the static and permissible cyclic stresses as

$\frac{1}{3}\left\{\left(P_{1}-P_{2}\right)^{2}+\left(P_{2}-P_{3}\right)^{2}+\left(P_{3}-P_{1}\right)^{2}\right\}^{\frac{1}{2}} \leq A-\alpha\left(S_{x}+S_{y}+S_{z}\right)$

where $P_{1,2,3}$ are the amplitudes of the alternating principal stresses, $S_{x, y, z}$ are the orthogonal static stresses, and $A$ and $\alpha$ are material constants. He suggested that the constants could be evaluated from uniaxial tests carried out at zero mean stress and with a zero to tension loading cycle.

If $\pm \sigma_{1}$ is the fatigue limit at zero mean stress, then

$$
S_{x}, S_{y}, S_{z}, P_{2}, P_{3}=0, \quad P_{1}= \pm \sigma_{1},
$$

therefore

$$
A=\frac{\sqrt{2}}{3} \sigma_{1}
$$

If $\sigma_{2} \pm \sigma_{2}$ is the fatigue limit under a zero to tensile stress loading cycle, then

$$
P_{2}, P_{3}, S_{y}, S_{z}=0, \quad S_{x}=\sigma_{2}, \quad P_{1}= \pm \sigma_{2},
$$

therefore

$$
A-\alpha \sigma_{2}=\frac{\sqrt{2}}{3} \sigma_{2},
$$

from which

$$
\alpha=\frac{\sqrt{2}}{3}\left(\frac{\sigma_{1}}{\sigma_{2}}-1\right) .
$$




\section{Conclusion}

These testing particulars imply that, with the exception of soft metals that deform noticeably subject to the applied loads, the fatigue limits of wrought metals and alloys represent part of the values predicted by the modified Goodman and Gerber expressions, the former providing the safer estimation.

There is a tendency for the data to lie closer to the modified Goodman line at low mean stresses and closer to the Gerber line at high mean stresses. It may well be for this reason that it has been reported that the data can be more accurately represented by the modified true-stress Goodman line, because this line lies above the conventional Goodman and Gerber lines at mean stresses that are a large fraction of the tensile strength. Materials which exhibit a definite fatigue limit give results somewhat closer to the Gerber line than to the Goodman line; materials which do not exhibit an $\mathrm{S} / \mathrm{N}$ curve having a sharp knee, such as high-strength aluminium alloys, give results falling around the modified Goodman line when the fatigue strength is estimated at relatively low endurances (about 107 cycles) but tend to approach the Gerber line when the fatigue strength is estimated at relatively long endurances (greater than 108 cycles).

In the case of all stated materials, the Sodeberg line renders a safe prediction, but in many occurrences it would be excessively safe.

It seems that, for ductile metals and alloys in which microcracks are launched and evolve in surface grains, the failure criterion applicable to an in-phase combined stress loading is some function of the cyclic maximum shear stresses, and the von Mises criterion is amply precise for practical intents.

In the case of in-phase combined bending and torsion, the results are well represented by an ellipse quadrant having as end-points the fatigue limits in pure bending and pure torsion. Little work has been done on the effect of out-of-phase combined stress loadings, but it is difficult to visualize their effect being more dangerous than when the corresponding loadings are applied in-phase. Combined bending and torsion fatigue tests in which there were various phase differences between the bending and torsion loading confirmed that in no case was the fatigue strength less than the corresponding in-phase case. The ratio of the fatigue limit in torsion to that in uniaxial loading is nearer 0.57 than 0.5 , which is probably due to the fact that there is a normal stress across the operative slip planes in the latter tests but not in the former. Thus, although the criterion for the onset of surface slip in a surface grain may indeed be that of maximum shear stress (as indeed it is for the onset of static slip in a single crystal), the progressive development of a microcrack will be easier when a normal stress acts across its faces than when it is absent, and thus the uniaxial fatigue limit is less than twice the torsional fatigue limit. The fact that this ratio approximates to that predicted by the von Mises criterion may therefore be co- incidental; indeed, it has been argued [6] that, although the strain energy or von Mises criterion is useful as a design formula, there is no evidence that fluctuating strain energy is a cause of fatigue cracking. In tests on materials not possessing a definite fatigue limit, the ratio of torsional to bending fatigue strengths increases progressively above the von Mises predicted value of 0.57 as the endurance at which the fatigue strengths are estimated decreases. This is presumably because it is easier for a crack having a normal cyclic stress acting across its faces to grow than for one which does not, thus making the bending fatigue strength (at a given endurance) increase less rapidly with decreasing endurance than the corresponding torsional fatigue strength. This fact may also account for the more marked effect, on both the uniaxial and torsional fatigue limit, of a uniaxial rather than a torsional mean stress, because the latter does not induce a normal stress component across the operative slip planes.

The effect of anisotropy does not appear to be important in interpreting combined bending and torsion fatigue tests, presumably because the fatigue limit in torsion is not significantly dependent on the direction from which specimens are cut from the stock material.

However, experiments on thin-walled tubes under in-phase pulsating internal pressure and longitudinal alternating uniaxial loads yield rather fluctuating results, as a rule attributed to anisotropy, as the circumferential fatigue limit is substantially lower than that in the longitudinal course.

\section{References}

[1] Frost, N. E., Marsh, K. J., Pook, L. P.: Metal Fatigue. Dover Publications, Inc. Mineola, New York, 1999, 499 p.

[2] Heywood, R. B.: Designing Against Fatigue. Chapman and Hall, London, 1962.

[3] Forrest, P. G.: Fatigue of Metals. Pergamon Press, Oxford, 1962.

[4] Findley, W. N., Mathur, P. N.: "Proc. Soc. Exp. Stress Analysis", 14, 35, 1956.

[5] Sines, G.: Metal Fatigue. Mc Graw - Hill, New York, 1959, $145 \mathrm{p}$.

[6] Findley, W. N., Mathur, P. N., Szcepanski, E., Temel, A. O.: "Trans. Am. Soc. Mech. Engrs. J. Bas. Eng.", 83, 10, 1961.

Doc. Ing. Petr Brož, DrSc.

phone: + 420224354630

e-mail: petr.broz@fsv.cvut.cz

Department of Concrete and Masonry Structures

Czech Technical University in Prague

Faculty of Civil Engineering

Thákurova 7

16629 Prague, Czech Republic 